\title{
CAN WE USE LOW-COST 360 DEGREE CAMERAS TO CREATE ACCURATE 3D MODELS?
}

\author{
L. Barazzetti ${ }^{1}$, M. Previtali ${ }^{1}$, F. Roncoroni ${ }^{2}$ \\ ${ }^{1}$ Dept. of Architecture, Built environment and Construction engineering (ABC) \\ Politecnico di Milano, Piazza Leonardo da Vinci 32, Milan, Italy \\ ${ }^{2}$ Polo Territoriale di Lecco, via Previati 1/c, Lecco \\ (luigi.barazzetti, mattia.previtali, fabio.roncoroni)@polimi.it \\ http://www.gicarus.polimi.it
}

Commission II / 8

KEY WORDS: $360^{\circ}$ camera, Accuracy, Automation, Low-cost, Orientation, Orthophoto, Surface reconstruction

\begin{abstract}
:
360 degree cameras capture the whole scene around a photographer in a single shot. Cheap 360 cameras are a new paradigm in photogrammetry. The camera can be pointed to any direction, and the large field of view reduces the number of photographs. This paper aims to show that accurate metric reconstructions can be achieved with affordable sensors (less than 300 euro). The camera used in this work is the Xiaomi Mijia Mi Sphere 360, which has a cost of about 300 USD (January 2018). Experiments demonstrate that millimeter-level accuracy can be obtained during the image orientation and surface reconstruction steps, in which the solution from $360^{\circ}$ images was compared to check points measured with a total station and laser scanning point clouds. The paper will summarize some practical rules for image acquisition as well as the importance of ground control points to remove possible deformations of the network during bundle adjustment, especially for long sequences with unfavorable geometry. The generation of orthophotos from images having a $360^{\circ}$ field of view (that captures the entire scene around the camera) is discussed. Finally, the paper illustrates some case studies where the use of a $360^{\circ}$ camera could be a better choice than a project based on central perspective cameras. Basically, $360^{\circ}$ cameras become very useful in the survey of long and narrow spaces, as well as interior areas like small rooms.
\end{abstract}

\section{INTRODUCTION}

Image-based modeling algorithms allow the automatic generation of $3 \mathrm{D}$ models from photographs. The commercial market offers several solutions for automated 3D modeling with a consolidated workflow: tie point extraction, bundle adjustment, dense image matching, surface reconstruction. Examples of software for close-range photogrammetry are Agisoft PhotoScan, PhotoModeler, ContextCapture, Pix4Dmapper, among the others.

Most applications in the photogrammetric domain are carried out using central perspective (pinhole) cameras, notwithstanding fisheye lenses are also becoming very popular for metric reconstructions. On the other hand, new 360 degree cameras are available on the commercial market. Some of these sensors have a limited cost (100-600 USD). 360-degree cameras capture the whole scene around a photographer in a single shot. Low-cost 360 cameras are becoming a new paradigm for photogrammetry. In fact, the camera can be pointed to any direction, and the large field of view reduces the number of photographs.

Images (at least 2) acquired from different points can be used to create a 3D model. Multiple images can be processed following the typical workflow for image processing based on the spherical (equirectangular) camera model. For some examples, the reader is referred to Strecha et al., (2015), Abate et al., (2017), Aghayaria et al. (2017), Barazzetti et al. (2017), Matzen et al. (2017). Other examples where the authors tried to survey narrow spaces with low-cost sensors (using the fisheye or the spherical camera model) were described by Fiorillo et al. (2016), Mandelli et al. (2017), Perfetti et al. (2017), Fassi et al. (2018). Table 1 shows some of the sensors with their average price in February 2018.

\begin{tabular}{|c|c|}
\hline 360 camera & $\begin{array}{c}\text { Average Price in } \\
\text { February 2018 (euro) }\end{array}$ \\
\hline Samsung Gear 360 & 90 \\
\hline Garmin VIRB 360 & 800 \\
\hline Insta 360 Air Voor & 140 \\
\hline Nikon KeyMission 360 & 350 \\
\hline Xiaomi Mijia Mi Sphere 360 & 220 \\
\hline LG 360 & 150 \\
\hline 360FLY & 750 \\
\hline Samsung New Gear 360 & 300 \\
\hline Ricoh Theta V & 430 \\
\hline Ricoh THETA S & 350 \\
\hline GoXtreme Dome 360 & 90 \\
\hline Ssstar & 100 \\
\hline Sansnail V1 & 65 \\
\hline YI VR 360 & 400 \\
\hline Motorola Moto 360 & 290 \\
\hline Gopro Odyssey & 12,200 \\
\hline Videostich Orah 4i & 2,900 \\
\hline Gopro Omni & 4,000 \\
\hline Nokia OZO & 49,000 \\
\hline Sphericam 2 & 1,350 \\
\hline Insta360 Pro & 3,600 \\
\hline
\end{tabular}

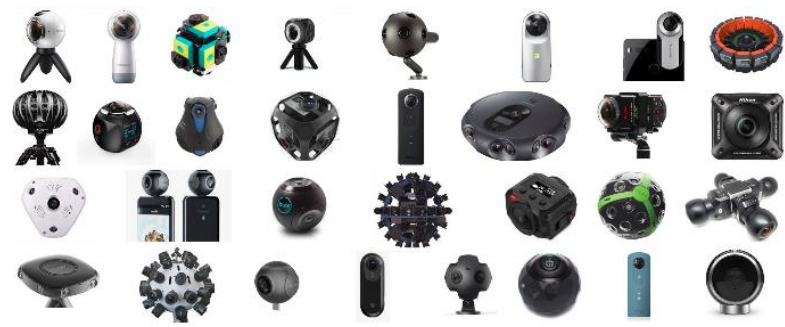

Table 1 . Some $360^{\circ}$ camera and their average price (February 2018). 
The camera used in this work is a Xiaomi Mijia Mi Sphere 360, which has a cost of about 300 USD. Such spherical images have a (max) resolution of $6912 \times 3456$ pixels and can be created by stitching set of front- and rear-facing images. The software for image stitching is Madventure 360 Camera, which is available for both mobile and desktop platforms. Preliminary camera calibration is not necessary because we assume that the final equirectangular image is a distortion-free projection.
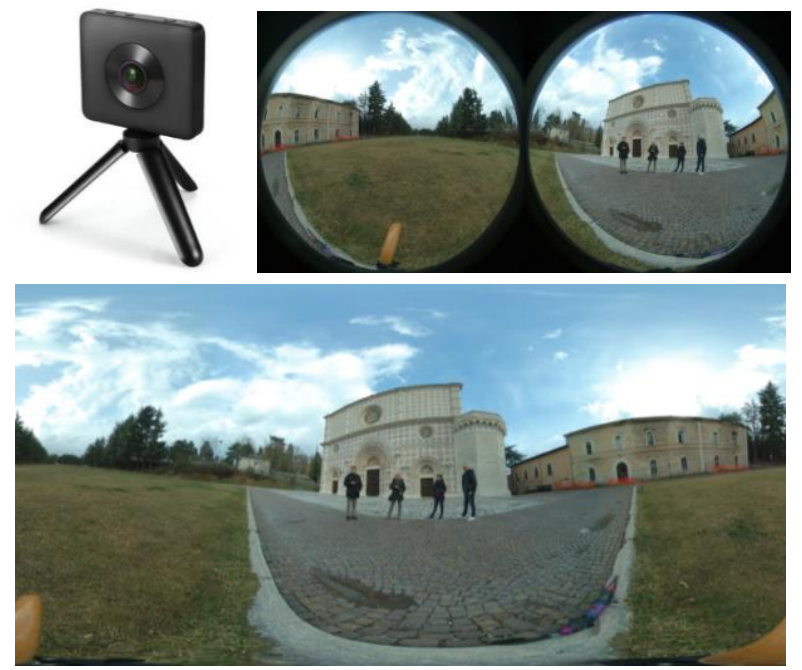

Figure. 1 The Xiaomi Mijia Mi Sphere 360 used in this work.

As mentioned, this paper aims to show that accurate metric reconstructions can be achieved with affordable sensors (less than 300 USD). The use of spherical images in photogrammetry is not new, notwithstanding most applications were carried out with images collected with a rotating camera and stitched with software for panorama generation. Fangi and Nardinocchi (2013) developed a mathematical formulation that turns pixel coordinates into horizontal and vertical angles measured with a theodolite. Then, bundle adjustment is similar to the adjustment of geodetic networks with an additional correction for the $\mathrm{Z}$ axis, which is not vertical. More details are reported in Fangi (2017), whereas several applications were then presented in Barazzetti et al. (2010), D'Annibale and Fangi (2009), Fangi (2007; 2009), Fangi and Pierdicca (2012), Pisa et al. (2010).

Recently, some commercial software have incorporated the spherical camera model. The software tested in this work are Agisoft PhotoScan and Pix4Dmapper, which can process such images and produce texturized meshes and orthophotos (Kwiatek and Tokarczyk, 2014; Kwiatek and Tokarczyk, 2015, Pérez Ramos and Robleda Prieto, 2015.)

\section{ACCURACY OF IMAGE ORIENTATION}

Metric accuracy evaluation of the Xiaomi Mijia Mi Sphere 360 was carried out with a set of $15360^{\circ}$ images and some targets measured with a total station Leica TS30 (angle precision 0.5', distance precision $0.6 \mathrm{~mm}$ ). Images were processed with both Agisoft PhotoScan and Pix4Dmapper, setting the camera model to spherical and measuring target manually in each image. Both software support the equirectangular (spherical) camera model. Targets were installed in an area with bad illumination conditions. The texture of surfaces is also quite bad. In all, 6 targets were used as control point and 11 were set as check points. Images were automatically oriented with the tie points extracted by automated matching strategies available in both software. An image of the computed camera poses is shown in Figure 2.
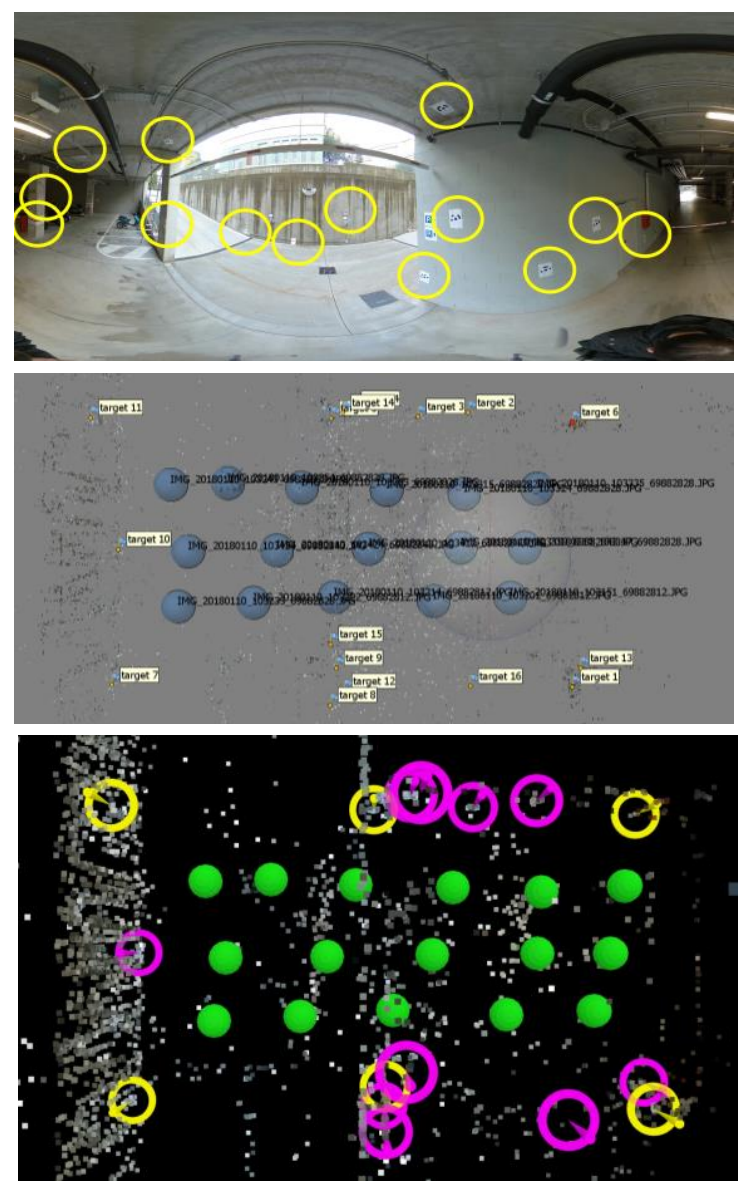

Figure 2. A spherical image of the test with the targets (top) and a vertical view of the test field after data processing with PhotoScan (middle) and Pix4D (bottom)

Green: camera station, Yellow: GCPs, Purple: Check Points

Statistics check points are illustrated in Table 2 and show an accuracy of about $6 \mathrm{~mm}$, whereas the test site is $10.9 \mathrm{~m} \mathrm{x} 6 \mathrm{~m} \mathrm{x}$ $3.7 \mathrm{~m}$. The relative accuracy achieved is about 1:2000.

\begin{tabular}{|c|c|c|c|}
\hline $\begin{array}{c}\text { 11 Check } \\
\text { points }\end{array}$ & $\begin{array}{c}R M S E X \\
(\mathrm{~mm})\end{array}$ & $\begin{array}{c}R M S E Y \\
(\mathrm{~mm})\end{array}$ & $\begin{array}{c}R M S E Z \\
(\mathrm{~mm})\end{array}$ \\
\hline PhotoScan & 6.8 & 7.2 & 6.7 \\
\hline Pix4Dmapper & 5.2 & 4.6 & 5.9 \\
\hline
\end{tabular}

Table 2. RMSE estimated on set of 11 check points.

Two additional considerations deserve to be mentioned. Figure 3 shows the camera locations and image overlap in PhotoScan (top), and the visualization of Pix4Dmapper with camera rays. As can be seen, a set of $360^{\circ}$ images with such configuration (e.g., the survey of a room) provides a large overlap. Tie points are visible in all the images. On the other hand, this does not mean that the software can match the same point in all the different images, notwithstanding the results obtained in this 
example reveal a very high multiplicity for the points (i.e., the number of images where the same point is visible).
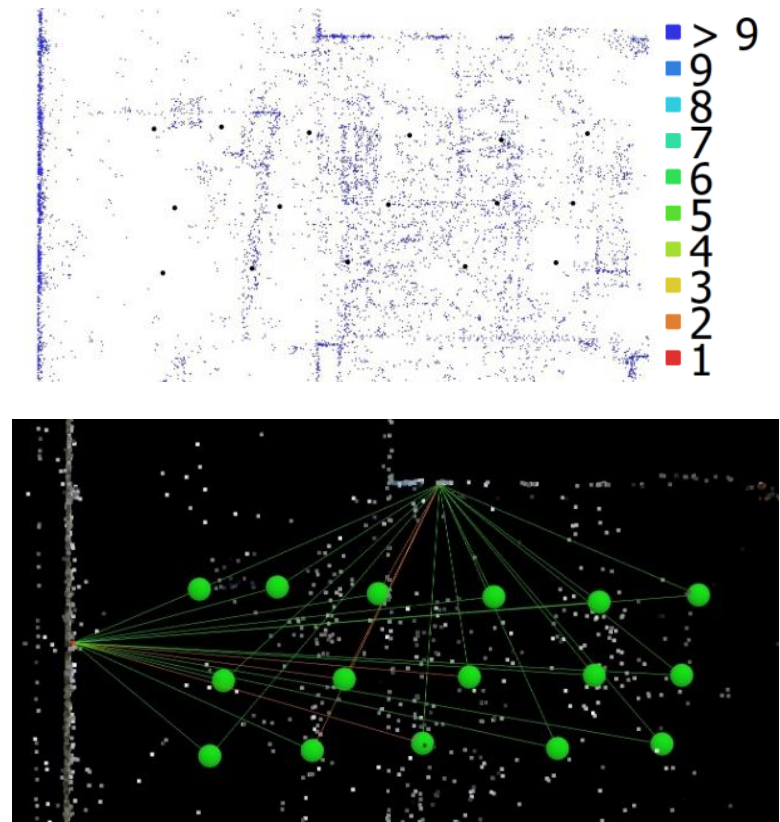

Figure 3. Spherical images provide a significant overlap. In this example, the same point is theoretically visible in all the images. However, this does not mean that the automatic extraction of tie points will provide matches visible in the whole dataset.

The second considerations are related to Figure 4. This picture was extracted from the orientation report of Pix4Dmapper. It provides a graphic visualization of error ellipses for camera locations, which is quite similar to that of geodetic networks. Indeed, spherical image bundle block adjustment is similar to network adjustment without distance measurements. As the camera is not leveled, 3 rotation angles are added in the mathematical formulation (the Z-axis in the camera centered reference system is not the vertical direction). On the other hand, the camera was almost horizontal during image acquisition. Experiments with the camera rotated along the $\mathrm{Z}$ axis did not reveal any particular issues during image orientation. On the contrary, tests with tilted images including a rotation of $90^{\circ}$ (roll) failed bundle adjustment. As the camera provides a $360^{\circ}$ visualization, there is no reason to rotate the camera vertically, unless the user is interested in the reconstruction only in elements such as the roof and ceiling. The overall recommendation is to avoid images acquired with tilted images unless the image block is made up of just tilted images.

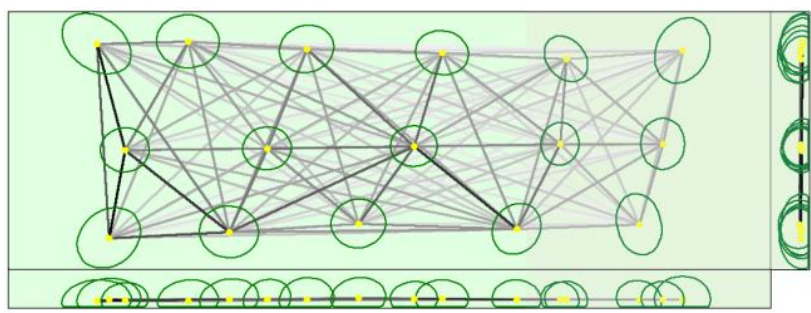

Figure 4. Such graphic representation with error ellipses is similar to the typical way to visualization of geodetic networks. The shape (circular or elongated) and size of ellipses provides an immediate indication of the quality of image orientation.

\section{ACCURACY OF DENSE POINT CLOUDS FROM SPHERICAL IMAGES}

A second dataset was acquired to evaluate the accuracy of the point cloud created from spherical images. In this case, a dataset of 15 spherical images was acquired and processed with PhotoScan (Figure 5, top). Pix4Dmapper was not used for this experiment because the trial version did not allow us to export the point cloud for further work in other software.
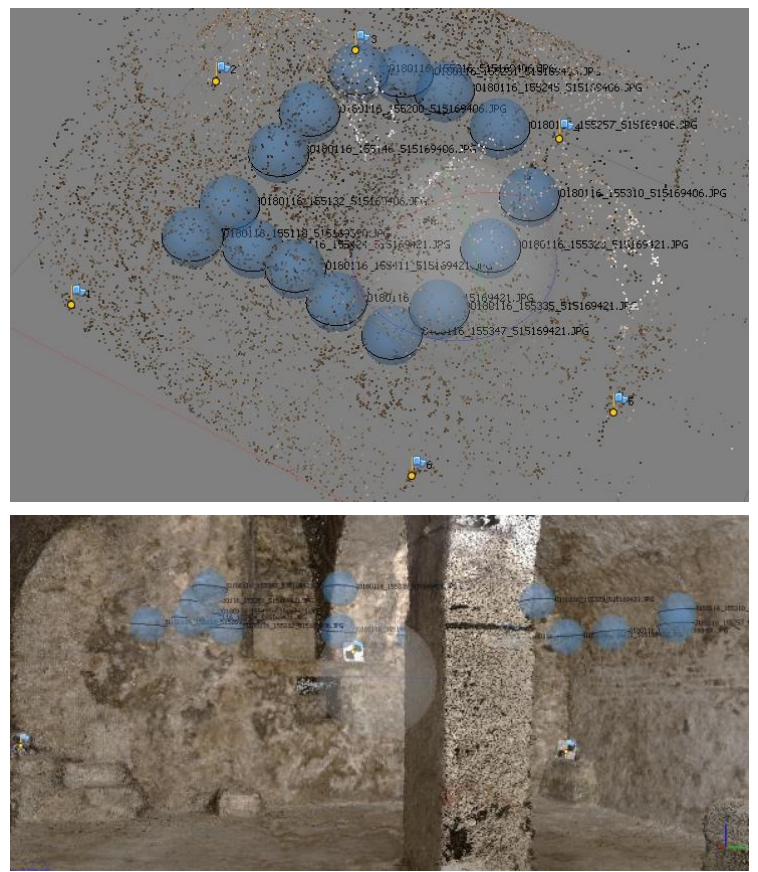

Figure 5. Orientation of images acquired in a cellar.

Dense image matching provided a point cloud that was compared to a reference dataset measured with a Faro Focus 3D laser scanner.

Data processing was carried out in a reference system provided by 6 targets (checkerboard) visible in both images and laser scans. This allowed the direct comparison between photogrammetric and laser scanning dataset, obtaining a discrepancy of about $5 \mathrm{~mm}$. Such comparison was carried out with CloudCompare getting the results shown in Figure 6.

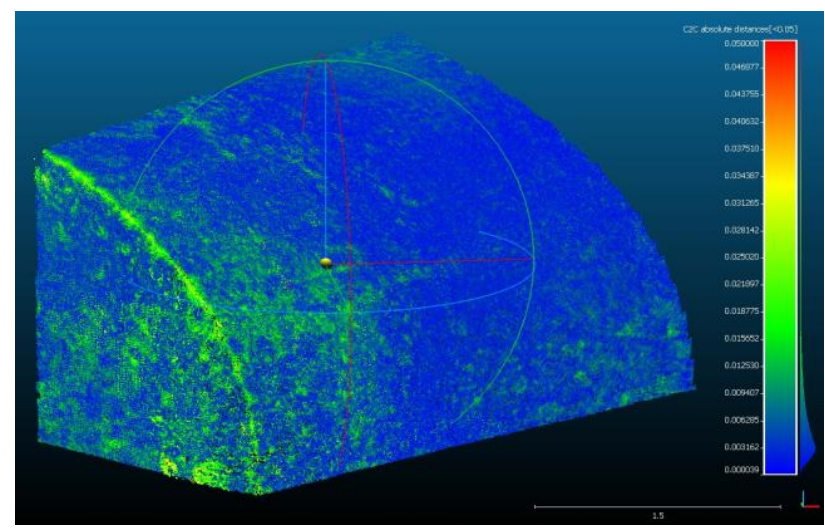

Figure 6. The discrepancy between the point cloud from 15 equirectangular projections and a laser scanning dataset used as reference. The error is about $5 \mathrm{~mm}$. The object shown is a part of a vaulted cellar. Other comparisons confirmed this metric accuracy. 


\section{ORTHOPHOTOS FROM SPHERICAL IMAGES}

The generation of orthophotos from images having with a $360^{\circ}$ field of view (that captures the entire scene around the camera) is an important issue. The variable ground sampling distance (GSD) has to be considered when multiple images are merged into a single mosaic. A test was carried out with a wall with a good texture (bricks) captured with both a central perspective camera (Nikon D610 with $20 \mathrm{~mm}$ lens) and the Xiaomi Mijia Mi Sphere 360. 6 targets measured with a total station were installed on the wall measured to provide a unique reference system for both projects.

Figure 7 (top) shows the results with the Nikon D610. The average camera object distance is $1.7 \mathrm{~m}$, and the GSD is 0.5 $\mathrm{mm}$. Some convergent images were added to obtain a better triangulation in space during bundle adjustment.

The same figure (bottom) shows the results with a set of 360 images. In this case, there is no reason to rotate the camera (horizontally), because it does not generate a better triangulation (the same ray would be available twice). Indeed, convergent images cannot be acquired rotating a spherical camera.

The average camera distance is $1.5 \mathrm{~m}$, and pixel size (estimated in the direction along the line that connects the camera center to the wall) is $1.3 \mathrm{~mm}$. This demonstrates that the resolution of 360 images is about 3 times worse (regarding GSD) than the resolution achievable with traditional images.
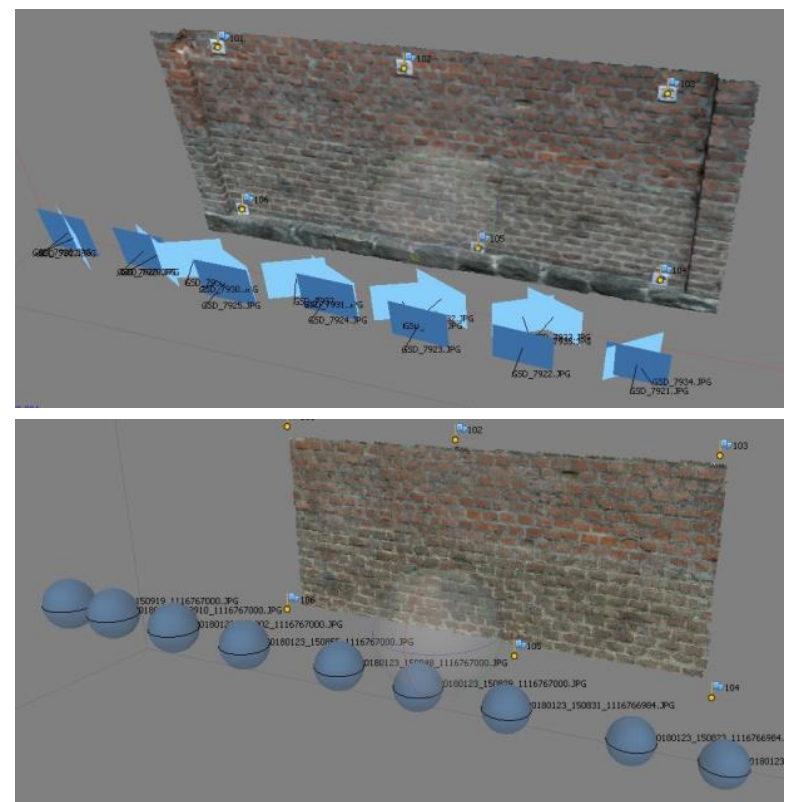

Figure 7. Reconstruction of a planar wall with pinhole and spherical cameras. In the case first case, convergent images provide a better triangulation in space. In the second one, there is no reason to rotate the camera.

The point clouds obtained through dense matching with PhotoScan were exported and analyzed in CloudCompare. The average discrepancy was $\pm 2.5 \mathrm{~mm}$, which is consistent with the average resolution of spherical images (Figure 8).

Finally, mesh and orthophoto were created for both projects. This means that PhotoScan provides tools to complete the entire photogrammetric workflow with spherical images. The orthophoto (Figure 9) confirms the previous considerations about image resolution. Orthophotos have a different resolution: $0.5 \mathrm{~mm}$ for the Nikon D610 (top), and $2 \mathrm{~mm}$ for the Xiaomi Mijia Mi Sphere. In this second case, although pixel resolution could also be set to $1.5 \mathrm{~mm}$, we preferred to use $2 \mathrm{~mm}$ for the variable GSD, which changes far from the image center.

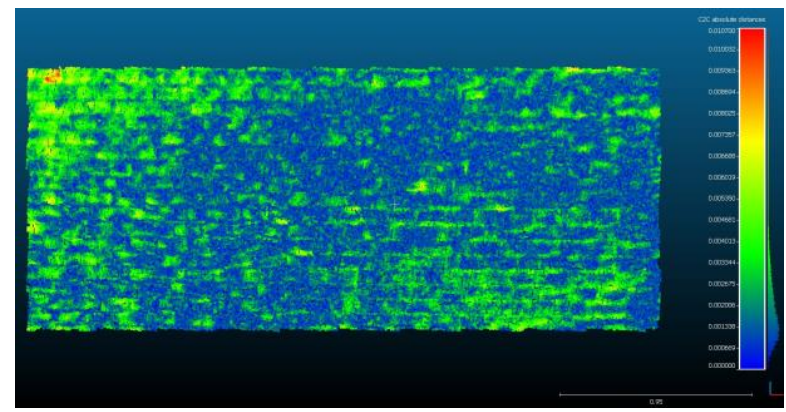

Figure 8. Comparison of the two point clouds in CloudCompare. The average discrepancy is $2.5 \mathrm{~mm}$.
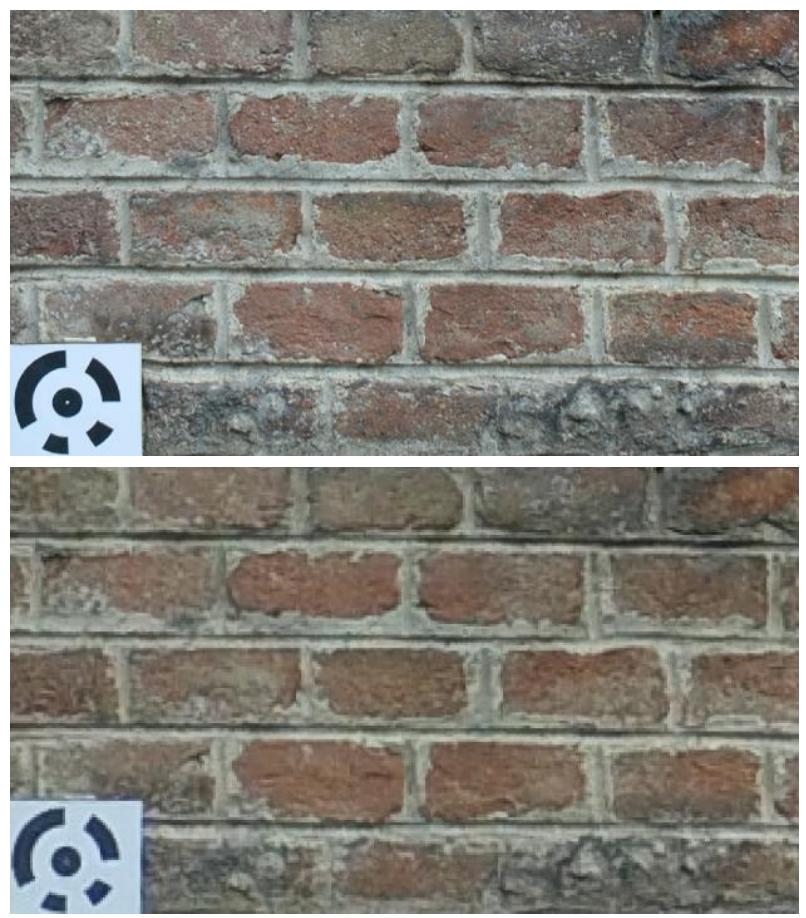

Figure 9. Orthophotos with pinhole (top) and spherical (bottom) images.

\section{WHY USE A SPHERICAL CAMERA?}

The results illustrated and discussed in the previous sections showed a good metric accuracy for the Xiaomi Mijia Mi Sphere 360 . In other words, metric accuracy reached \pm 1 pixel regarding image coordinates. The reader should pay particular attention that the high geometric resolution of such camera (24 megapixels) has to be considered on a $360^{\circ} \times 180^{\circ}$ field of view, resulting in a ground sampling distance (GSD) significantly lower than images acquired with traditional central perspective cameras.

It is the authors' opinion that such camera can be a valid tool for some specific applications. In some cases, spherical images are a better choice than traditional and fisheye images. For instance, such camera becomes very useful in the survey of long and narrow spaces, as well as interior areas like small rooms. Some examples are shown in Figure 10. The first case (Basilica) shows an image sequence acquired to model the three naves of a basilica. 

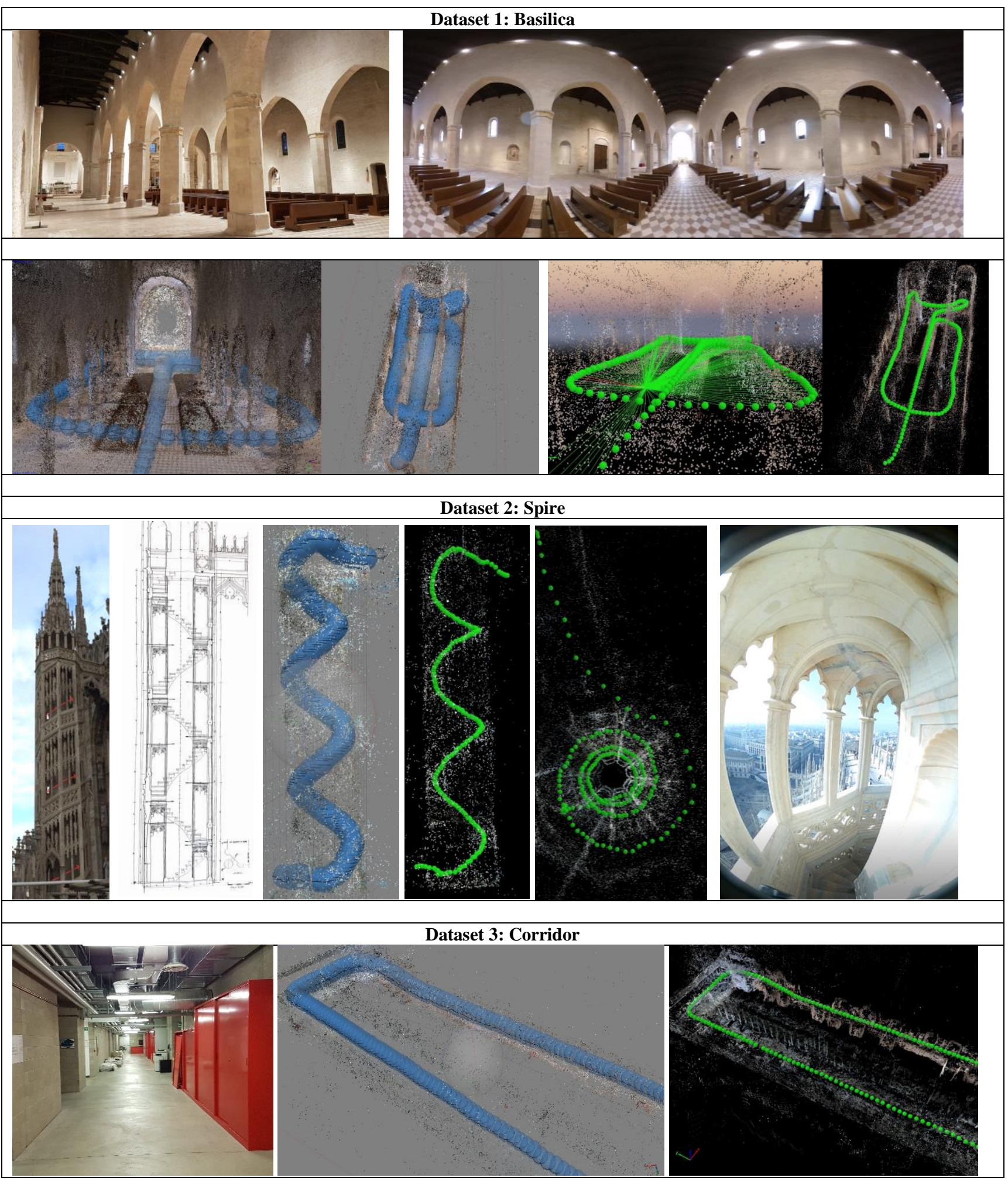

Figure 10. Some projects where the use of a $360^{\circ}$ camera could be a better choice than a traditional central perspective camera. 
The sequence is made up of 237 images, which were oriented with both PhotoScan (left) and Pix4Dmapper (right). Data collection took only a few minutes, whereas the same Basilica scanned with a laser scanner would require several hours. Apparently, the quality of the final point cloud from images would be not comparable with a laser scanning point cloud (regarding precision and density). On the other, the proposed method with spherical images would be a better solution for rapid documentation.

Another interesting example is the narrow staircase inside the spire (Figure 10, middle). Here, the limited space available makes spherical images a valid alternative to laser scanning and more traditional photogrammetric projects. Data acquisition took a few minutes and images were correctly oriented. In fact, it is possible to compare the shape of the staircase available in technical drawings. We did not carry out further data processing (dense image matching) because the aim was only to investigate the correctness of the reconstruction after image triangulation. Probably, the material (marble) would prevent the creation of a good point cloud. On the other hand, this example proves that spherical images could be a powerful tool for long and narrow spaces.

Finally, a sequence acquired in a narrow corridor was oriented with both software. The dataset is made up of more than 200 images. Images were successfully oriented in a very short data processing time (minutes). The same work carried out with traditional photogrammetry or (static) laser scanning would require a much longer data acquisition time.

Basically, the previous examples have demonstrated that data processing is feasible for large blocks. The geometry of a spherical image is more suitable than central perspective imaging techniques regarding the field of view and image overlap. On the other hand, very long sequences could result in accuracy problems, especially when multiple images are progressively added without external constraints. The use of ground control points measured with a total station remains a primary tool to control network geometry, especially for long sequences such as the spire and corridor sequences. The first sequence has instead several points matched on images acquired along different strips. Such solution probably provides better results regarding metric accuracy also for adjustment processes with minimal constraints.

Other issues that have to be considered during image acquisition concern the illumination conditions, which are difficult to control in the case of spherical shots.

\section{CONCLUSIONS}

The different tests proposed in this paper reveal a remarkable potential of the Xiaomi Mijia Mi Sphere 360 for photogrammetric applications. The metric accuracy achieved with the Xiaomi Mijia Mi Sphere 360 is in the range $0.5-1.5$ pixels, that is also consistent with typical results of more traditional photogrammetric projects based on central perspectives. On the other hand, the user has to consider the large field of view of equirectangular images: 24 megapixels on a 360 field of view is not a very high resolution. The GSD of a spherical project is 4-6 times worse than the same project with central perspective lenses. Other problems were found when the scene has inhomogeneous lighting conditions, which are difficult to correct.

A comparison of such results with the work proposed by Barazzetti et al. (2017), in which the authors tested another 360 camera (Samsung Gear 360), reveal a relevant improvement of $3 \mathrm{D}$ point precision and completeness of the model.
One could say that after one year, a new camera with a similar price provided much better results for photogrammetric applications. Apparently, this is just a general consideration. On the other hand, it is the authors' opinion that new $360^{\circ}$ cameras with better features will be available soon. This also opens new opportunities for photogrammetric projects of long and narrow spaces such as those illustrated in this paper.

\section{REFERENCES}

Abate, D., Toschi, I., Sturdy-Colls, C., and Remondino, F., 2017. A low-cost panoramic camera for the $3 \mathrm{~d}$ documentation of contaminated crime scenes. Int. Arch. Photogramm. Remote Sens. Spatial Inf. Sci., XLII-2/W8, 1-8.

Aghayaria, S., Saadatsereshta, M., Omidalizarandi, M., Neumannb, I., 2017. Geometric Calibration of Full Spherical Panormamic Ricoh-Theta Camera. In: ISPRS Annals of the Photogrammetry, Remote Sensing and Spatial Information Sciences, Vol. 4(1/W1), pp. 237-245.

Barazzetti, L., Fangi, G., Remondino, F. and Scaioni, M., 2010. Automation in multi image spherical photogrammetry for $3 \mathrm{D}$ architectural reconstructions. 11th International Symposium on Virtual Reality, Archaeology and Cultural Heritage (VAST), Paris, France. 75-81 (on CD-ROM).

Barazzetti, L., Previtali, M., Roncoroni, F., 2017. 3D Modelling with the Samsung Gear 360. In: The Int. Archives of Photogrammetry, Remote Sensing and Spatial Information Sciences, Vol. 42(2/W3), pp. 85-90

D'Annibale, E. and Fangi, G., 2009. Interactive modelling by projection of oriented spherical panorama. International Archives of Photogrammetry, Remote Sensing and Spatial Information Sciences, 38(5/W1). 6 pages (on CD-ROM).

Fangi, G., 2007. The multi-image spherical panoramas as a tool for architectural survey. International Archives of Photogrammetry, Remote Sensing and Spatial Information Sciences, 36(5/C53): 311-316.

Fangi, G., 2009. Further developments of the spherical photogrammetry for cultural heritage. XXII International Committee for Cultural Heritage (CIPA), Kyoto, Japan. 6 pages (on CD-ROM).

Fangi, G., 2010. Multi scale, multi resolution spherical photogrammetry with long focal lenses for architectural survey. ISPRS Midterm Symposium, Newcastle, UK. 228-233 (on CDROM).

Fangi, G. and Pierdicca, R., 2012. Notre Dame du Haut by spherical photogrammetry integrated by point cloud generated by multi view software. International Journal of Heritage in the Digital Era, 1(3): 461-479.

Fangi, G., 2017. The book of spherical photogrammetry: Theory and experiences. Edizioni Accademiche Italiane, 300 pages.

Fassi F., Troisi S., Baiocchi V., Del Pizzo S., Giannone F., Barazzetti L., Previtali M., Polari C., Perfetti L., Roncoroni F., 2018. Fisheye Photogrammetry to Survey Narrow Spaces in Architecture and a Hypogea Environment. In Latest Developments in Reality-Based 3D Surveying and Modelling, MDPI Books, DOI: 10.3390/books978-3-03842-612-7/2.

Fiorillo, F., Limongiello, M., and Fernández-Palacios, B. J., 2016. Testing GoPro for 3D model reconstruction in narrow spaces. Acta Imeko, 5, 64-70. 
Kwiatek K., Tokarczyk R., 2014. Photogrammetric Applications of Immersive Video Cameras. ISPRS Annals of Photogrammetry, Remote Sensing and Spatial Information Sciences, vol. 1, pp. 211-218.

Kwiatek, K., Tokarczyk, R., 2015. Immersive Photogrammetry in 3D Modelling. Geomatics and Environmental Engineering, Volume 9, Number 2, pp. 51-62.

Matzen, K., Cohen, M. F., Evans, B., Kopf, J., Szeliski, R., 2017. Low-Cost 360 Stereo Photography and Video Capture. In: Journal ACM Transactions on Graphics, Vol.36(4), pp. 148.

Mandelli, A., Fassi, F., Perfetti, L., and Polari, C., 2017. Testing Different Survey Techniques To Model Architectonic Narrow Spaces, Int. Arch. Photogramm. Remote Sens. Spatial Inf. Sci., XLII-2/W5, 505-511.

Pérez Ramos, A., Robleda Prieto, G., 2015. Only image based for the $3 \mathrm{~d}$ metric survey of gothic structures by using frame cameras and panoramic cameras. The International Archives of the Photogrammetry, Remote Sensing and Spatial Information Sciences, Volume XLI-B5, 2016 XXIII ISPRS Congress, 12-19 July 2016, Prague, Czech Republic, 363-370.

Perfetti, L., Polari, C., and Fassi, F., 2017. fisheye photogrammetry: tests and methodologies for the survey of narrow spaces. Int. Arch. Photogramm. Remote Sens. Spatial Inf. Sci., XLII-2/W3, 573-580.

Pisa, C., Zeppa, F. and Fangi, G., 2010. Spherical photogrammetry for cultural heritage. Proceeding of the Second Workshop on eHeritage and Digital Art Preservation, Florence, Italy. 3-6.

Strecha, C., Zoller, R., Rutishauser, S., Brot, B., SchneiderZapp, K., Chovancova, V., and Glassey, L., 2015. Quality assessment of 3D reconstruction using fisheye and perspective sensors. ISPRS Annals of the Photogrammetry, Remote Sensing and Spatial Information Sciences, 2(3), 215.

Agisoft PhotoScan: http://www.agisoft.com

Pix4Dmapper: https://pix4d.com/

CloudCompare: http://www.danielgm.net/cc/ 